\title{
Effect of Bimodal Harmonic Structure Design on the Deformation Behaviour and Mechanical Properties of Co-Cr-Mo Alloy
}

Sanjay Kumar VAJPAI ${ }^{\mathrm{a} *}$, Choncharoen SAWANGRAT ${ }^{\mathrm{b}}$, Osamu YAMAGUCHI ${ }^{\mathrm{c}}$, Octav Paul CIUCA ${ }^{\mathrm{d}}$,

\author{
Kei AMEYAMA ${ }^{\mathrm{c}}$
}

${ }^{a}$ Research Organization of Science and Technology, Ritsumeikan University, 1-1-1 Noji-higashi, Kusatsu, Shiga 525-8577, Japan. Email: vajpaisk@ gmail.com

${ }^{\mathrm{b}}$ Department of Industrial Engineering, Faculty of Engineering, Chiang Mai University, Chiang Mai 50200, Thailand. Email: choncharoen@step.cmu.ac.th

${ }^{c}$ Department of Mechanical Engineering, College of Science and Engineering, Ritsumeikan University, 1-1-1 Noji-higashi, Kusatsu, Shiga 525-8577, Japan. Email: rm0011xh@ed.ritsumei.ac.jp; ameyama@se.ritsumei.ac.jp

d A14, Materials Science Centre, University of Manchester, Manchester, M1 7HS, England. Email: octav.ciuca@manchester.ac.uk

*Corresponding author: Department of Mechanical Engineering, Ritsumeikan University (Biwako Kusatsu Campus), 1-1-1 Noji-higashi, Kusatsu, Shiga, 525-8577, Japan. Tel.: +81 77561 2749; fax: +81 77561 2665. E-mail: vajpaisk@ gmail.com 


\begin{abstract}
In the present work, Co-Cr-Mo alloy compacts with a unique bimodal microstructural design, harmonic structure design, were successfully prepared via a powder metallurgy route consisting of controlled mechanical milling of pre-alloyed powders followed by spark plasma sintering. The harmonic structured Co-Cr-Mo alloy with bimodal grain size distribution exhibited relatively higher strength together with higher ductility as compared to the coarse-grained specimens. The harmonic Co-Cr-Mo alloy exhibited a very complex deformation behavior wherein it was found that the higher strength and the high retained ductility are derived from fine-grained shell and coarse-grained core regions, respectively. Finally, it was observed that the peculiar spatial/topological arrangement of stronger fine-grained and ductile coarse-grained regions in the harmonic structure promotes uniformity of strain distribution, leading to improved mechanical properties by suppressing the localized plastic deformation during straining.
\end{abstract}

Keywords: Co-Cr-Mo alloy; Powder metallurgy; Harmonic structure; Bimodal grain structure; Deformation mechanism; Severe plastic deformation

\title{
1. Introduction
}

The Co-Cr-Mo alloys are an important class of structural biomaterials which are widely used for a variety of biomedical applications, such as coronary stent, dental implants and hip and knee replacements, due to their good biocompatibility and low radiolucency together with excellent mechanical properties and resistance to wear and corrosion [1-2]. Moreover, it has been demonstrated that the Co-Cr-Mo alloys are also suitable for aerospace bushings and bearing applications [3]. Therefore, in view of the increasing 
demand of Co-Cr-Mo alloys for these important practical applications, continuous efforts are being made for further strengthening to improve the performance and reliability of these alloys in service wherein achieving high strength together with sufficient ductility is a critical issue. Grain refining is a well-known approach to achieve high strengths in metallic materials [4-9]. However, grain refinement results in significantly reduced ductility as compared to coarse-grained bulk materials. A similar trend has also been observed in case of grain-refined Co-Cr-Mo alloys [10-12]. Therefore, in order to achieve higher toughness and improved performance, further efforts are required to prepare Co-Cr-Mo material with higher strength without sacrificing ductility. In recent years, it has been demonstrated that materials with bimodal grain size distribution exhibit high strength together with high ductility, wherein ultrafine grains provide increased strength whereas coarse grains ensure higher retained ductility [13-19]. However, interestingly, no extensive research reports were found in the literature on the strengthening of Co-Cr-Mo alloys through creation of bimodal structure.

There are several issues associated with the control of bimodal microstructure when preparing bulk metals and alloys through conventional processing approach consisting of severe plastic deformation and controlled annealing [13-16]. On the other hand, powder metallurgy based approach was found to be suitable to prepare bulk metals and alloys with a tailored bimodal microstructure [19-23]. Recently, Ameyama and coworkers proposed an exquisite microstructural design with bimodal grain size distribution, called "harmonic structure", and a powder metallurgy processing approach to prepare bulk materials with such controlled bimodal microstructures [24-31]. An schematic of the proposed microstructural design and procedure to create the same is 
illustrated in Figure 1. In harmonic structure, coarse-grained regions ("core") are enclosed in a continuously connected three-dimensional network of regions with fine-grained structure ("shell"). A variety of metal and alloys with harmonic structure were prepared, wherein it was demonstrated that harmonic structure design leads to high strength together with retained high ductility.

Recently, a preliminary work was carried out by the present authors to prepare bimodal Co-Cr-Mo alloys with harmonic structure design and evaluate their properties [32]. However, the previous work did not include the detailed studies on the microstructural characteristics of the as-prepared harmonic Co-Cr-Mo alloy and microstructural evolution during subsequent plastic deformation. Therefore, it is not clear as to how the harmonic structure design results in such an excellent combination of strength and ductility in Co-Cr-Mo alloy. Also, there have been no studies to assess the relative advantage/disadvantage of harmonic structured over conventional heterogeneous bimodal microstructure in Co-Cr-Mo alloys. It would also be worth mentioning that the plastic deformation leads to strain-induced $\alpha$-fcc to $\varepsilon$-hcp phase transformation and deformation twinning in Co-Cr-Mo alloys. It is envisaged that such microstructural changes would have a crucial effect on the deformation behavior and resulting mechanical properties, owing to the presence of bimodal grain size distribution. Therefore, the present work aims to prepare Co-Cr-Mo alloy compacts with a unique bimodal microstructural design, harmonic structure design, via a powder metallurgy route consisting of controlled mechanical milling of pre-alloyed powders and spark plasma sintering. The microstructural characteristics of the as-prepared harmonic Co-Cr-Mo alloy and the microstructural evolution with increasing degree of deformation has been investigated and elaborated. Subsequently, results related with the 
effect of harmonic structure design and its interplay with the microstructural changes, such as strain-induced $\alpha$-fcc to $\varepsilon$-hcp phase transformation, on the deformation behavior and resulting properties of the Co-Cr-Mo alloy has been presented and discussed. Finally, results related with the mechanical properties and deformation behavior of conventional heterogeneous bimodal Co-Cr-Mo compacts are presented and compared to understand the advantages of harmonic structure design over conventional heterogeneous bimodal structures.

\section{Experimental procedure}

In this present work, pre-alloyed powders of Co-Cr-Mo alloy (ASTM-F75 grade) has been used. The powder was prepared using Plasma Rotating Electrode Process (PREP) and the chemical composition of the powder is presented in Table 1. As-received spherical PREP powder had an average particle size approximately $217 \mu \mathrm{m}$. The as-received powder was mechanically milled under Argon atmosphere at room temperature using planetary ball mill, with stainless steel balls and vials, operating at a speed of $200 \mathrm{rpm}$ and ball-to-powder weight ratio 2:1. The powders were consolidated via Spark Plasma Sintering (SPS) in vacuum atmosphere at $1323 \mathrm{~K}$ under an applied pressure of 50MPa to prepare full density compacts. The milling and sintering time was adjusted based on the results of our previous preliminary work [32]. In order to prepare compacts, having well defined harmonic structure with approximately 60 volume $\%$ of fine grained area, powder milled for $54 \mathrm{ks}$ was used. On the other hand, to prepared compacts with conventional heterogeneous bimodal grain size distribution, powder milled for $180 \mathrm{ks}$ were mixed with un-milled powder in 1:1 weight ratio. To achieve near-full density, the holding time during sintering for un-milled PREP initial powders 
was kept $7.2 \mathrm{ks}$ whereas it was $3.6 \mathrm{ks}$ for mechanically milled powder as well as for the 1:1 weight ratio mixture of un-milled and milled powders. The microstructural characterization of the powders and sintered compacts was carried out using Scanning Electron Microscope equipped with Electron Backscattered Diffraction (EBSD) facility. The microstructural changes were also monitored by X-ray diffraction (XRD) method. Mechanical properties of the bulk specimens were evaluated by hardness measurements and tension tests at room temperature. Vickers micro-hardness was estimated by indentation under a load of $980.7 \mathrm{mN}$ and holding time of $5 \mathrm{~s}$. The hardness measurements were carried out randomly, wherein the distance between the indents was kept at least three times the size of the indents. Tensile properties were evaluated by carrying out tensile tests at a strain rate of $5.6 \times 10^{-4} \mathrm{~s}^{-1}$ using specimens with gauge size $3 \mathrm{~mm}$ X $1 \mathrm{~mm}$ X $1 \mathrm{~mm}$ The relative fraction of $\varepsilon$-HCP phase (wt\%) were determined through X-ray diffraction using the method proposed by Sage and Guillaud [33].

\section{Results and Discussion}

\subsection{Microstructural Characteristics of Harmonic Co-Cr-Mo Compacts}

The sintered compacts prepared from un-milled initial PREP Co-Cr-Mo powders (henceforth referred as "IP compacts") exhibited mostly homogeneous coarse grained (CG) structure with average grain size approximately $36.8 \mu \mathrm{m}$. Figure 2 shows the microstructure of the sintered compacts prepared from milled PREP Co-Cr-Mo alloy powders. It can be noted that the milled PREP powders resulted in sintered compacts with bimodal microstructure with equiaxed grains. However, it is interesting to note that fine- and coarse-grained regions have a unique topological distribution wherein the region with fine-equiaxed grains (approximately $60 \mathrm{vol} \%$, average grain size $=3.2 \mu \mathrm{m}$ ) 
form a continuous connected three-dimensional network which surrounds the coarse-grained regions (average grain size $=37.2 \mu \mathrm{m}$ ). This unique microstructural characteristic has been referred as "Harmonic" structure design. It would be worth mentioning that the regions with finer grains and coarser grains correspond to the severely deformed "shell" and relatively un-deformed "core" regions of the milled powders, respectively. Therefore, the fine-grained and coarse-grained areas will also be referred as "shell" and "core", respectively. These compacts would be referred as "harmonic" in the subsequent discussions. The presence of the equiaxed fine-grained structure in the shell region is an evidence of the fact that the recrystallization and grain growth processes were operative in the severely deformed outer sub-surface region of the milled powders. Figure 3 illustrates the XRD pattern of the harmonic Co-Cr-Mo alloy compacts, wherein it can be noticed that the compacts with harmonic structure consisted of primarily $\alpha$ phase together with only a small amount of martensitic $\varepsilon$ phase. Figure 4 shows the image quality (IQ) map and phase map, as obtained by EBSD, illustrating the microstructure and phase distribution in the harmonic $\mathrm{Co}-\mathrm{Cr}-\mathrm{Mo}$ alloy compacts. It can be clearly observed that the shell region consisted of fine-sized (of the order of 2-5 $\mu \mathrm{m}$ ) equiaxed grains wherein a number of fine-equiaxed grains exhibit the presence of a significant amount of annealing twins (Figure 4a). Moreover, the phase distribution map shows that the harmonic Co-Cr-Mo alloy consisted of primarily $\alpha$ phase whereas martensitic $\varepsilon$ phase is present only in a small quantity (Figure 4b). These results are in conformity with the XRD results as shown in Figure 3. However, interestingly, most of the $\varepsilon$ phase was found to be present only in the fine-grained shell area whereas coarse-grained core remains more or less free from the presence of martensitic $\varepsilon$ phase. The presence of $\varepsilon$-hcp phase in the shell regions can be attributed 
primarily to the retention of deformation induced martensite in the milled powders, together with a possible formation of plastic-deformation induced martensite due to applied pressure during cooling stage of spark plasma sintering [32, 34].

\subsection{Mechanical Properties and Deformation Behavior of Harmonic Co-Cr-Mo}

\section{Alloy Compacts}

Figure 5 shows representative nominal stress-nominal strain curves of the specimens prepared from IP and harmonic compacts. It can be observed that coarse-grained IP as well as bimodal harmonic exhibited a trend of continuously increasing strength with increasing elongation until fracture without showing any sign of existence of point of plastic instability, i.e. necking. This trend is very much peculiar to Co-Cr-Mo alloys which undergo continuous strain-hardening with increasing degree of plastic deformation. However, it can be noted that the specimens with harmonic structure clearly exhibited relatively higher strength, both yield strength and tensile strength, and ductility as compared to the coarse-grained IP specimens. Moreover, it is also interesting to note that the increment in the tensile strength and ductility, as demonstrated by fracture stress and strain-to-fracture, was considerably higher as compared to that observed for yield strength. Therefore, these results clearly indicate that the formation of harmonic structure led to improved mechanical properties in Co-Cr-Mo alloy also, which is similar to the results found for other materials in our previous studies [24-31].

A closer view at the initial part of stress-strain curve, as shown in Figure 6, reveals that the strain hardening rate in the initial stages of the deformation of the harmonic specimens was significantly higher as compared to that of the coarse-grained IP specimens. However, the strain hardening rate during subsequent deformation stages 
was more or less similar for both types of specimens. From literature, it has been observed that the decreasing grain size leads to not only higher strengths but also an increasing strain hardening rate in the early stages of deformation in Co-Cr-Mo alloys $[10,35-36]$. However, the ductility was found to decrease with decreasing grain size. Therefore, a significant improvement in the mechanical properties of harmonic structure Co-Cr-Mo alloy appears to be associated with the unique microstructural design wherein early stages of deformation are governed by the interconnected fine-grained shell region. Hence, systematic study was carried out on the deformation behavior of Co-Cr-Mo alloy specimens with harmonic structure.

In order to understand the deformation behavior of harmonic structured material, specimens were deformed up to $10 \%$ and $20 \%$ by straining under quasi-static tensile loading and the microstructure and properties of these deformed/strained specimens were compared with those of the un-strained/un-deformed specimens. Figure 7 shows the XRD patterns of the un-deformed and deformed harmonic Co-Cr-Mo alloy specimens, illustrating the microstructural evolution with increasing degree of deformation. It can be clearly observed that the harmonic Co-Cr-Mo alloy specimens without plastic straining consist of primarily $\alpha$-fcc phase together with small amount of $\varepsilon$-hcp phase. However, the intensity of the peaks corresponding to martensitic $\varepsilon$-hcp phase increased with increasing amount of applied strain, i.e. after $10 \%$ and $20 \%$ applied strain. These results indicate that the deformation induced $\alpha$-fcc to $\varepsilon$-hcp phase transformation occurred as a result of tensile straining, and the amount of martensitic $\varepsilon$-hcp phase also increased with increasing degree of deformation. The formation of martensitic $\varepsilon$-hcp phase due to strain-induced $\alpha$-fcc to $\varepsilon$-hcp phase transformation is a typical characteristic of Co-Cr-Mo alloys when subjected to plastic deformation at room 
temperature [37].

Figure 8 shows the variation of $\varepsilon$-hcp phase with increasing tensile straining in the harmonic Co-Cr-Mo alloy specimens, as estimated using XRD results. For comparison, the variation of $\varepsilon$-hcp phase for the coarse-grained IP compacts is also shown in Figure 8. It can be observed that, in case of harmonic structured specimens, the amount of $\varepsilon$-hcp phase increased significantly with increasing degree of deformation. However, it is interesting to note that a significant amount of $\varepsilon$-hcp phase was formed in the very early stages of deformation, i.e. approximately within initial $2 \%$ of the total strain. In subsequent stages of deformation, the phase transformation rate was relatively lower as compared to that of the early stages of deformation. On the contrary, in case of coarse-grained IP specimens, there was only a very small increment in the amounts of $\varepsilon$-hcp phase with increasing plastic straining and it remained considerably lower as compared to that of the harmonic structured specimens. These results clearly suggest that the harmonic structure design leads to a different deformation mechanism, as compared to coarse-grained material, resulting in the improved mechanical properties. In particular, the early stages of deformation appear to be significantly different as compared to that of its coarse-grained counterpart.

Figure $9 \mathrm{a}, 9 \mathrm{~b}$ and $9 \mathrm{c}$ shows the EBSD-GB images of a harmonic structured Co-Cr-Mo alloy specimen after $0 \%, 10 \%$, and $20 \%$ tensile deformation, respectively, whereas, Figure $9 \mathrm{~d}, 9 \mathrm{e}$, and $9 \mathrm{f}$ demonstrate the evolution and distribution of $\varepsilon$-hcp phase in the area shown in 9a, 9b and 9c, respectively. It would be worth mentioning that the same area was observed on the specimen after progressive deformation strains. Clearly, Figure 9 illustrates the microstructural evolution and distribution of $\varepsilon$-hcp with increasing plastic strain. Similar to Figures 7 and 8, it can be clearly observed that the 
$\varepsilon$-hcp phase increased with increasing degree of deformation. However, it is interesting to note that most of the $\varepsilon$-hcp phase evolved in the fine-grained shell region, and only a small amount of strain induced $\varepsilon$-hcp phase was formed in the coarse-grained core region. Figure 10 shows an enlarged view of the microstructure and phase distribution in the deformed ( 20\%) harmonic Co-Cr-Mo alloy specimens. Figure 10a and 10b shows the EBSD-IQ image and its corresponding phase distribution map, respectively. It can be clearly observed that a large plastic deformation led to formation of a large number of intra-granular striations (marked by arrows in Figure 10a), twins, and a significant amount of $\varepsilon$-hcp phase in the harmonic structured specimens. Particularly, coarse-grained core region exhibited relatively larger density of intra-granular striations, which extended across the whole grain. On the other hand, the twinning and formation of $\varepsilon$-hcp was more significant in the fine-grained shell region as compared to the coarse-grained core region. These evidences suggest that the both coarse- and fine-grained regions underwent severe plastic deformation with slightly different mode of deformation.

It appears that fine-grained shell region was more susceptible to undergo phase transformation as compared to the coarse-grained core region, under a given plastic deformation. Generally, it is well known that the martensitic transformation in Co-Cr alloys occurs via athermal (AT), isothermal, and strain induced transformation (SIT) owing to the low stacking fault energies (SFEs) associated with this class of alloys [35, 37-40]. Particularly, in the case of SIT, it was observed that the plastic deformation induces several structural defects, such as shear bands and stacking faults. These defects provide sites for the nucleation of martensitic $\varepsilon$-phase, especially at the twin boundaries $[34,41-42]$. Since the fine-grained shell regions had a larger number of annealing twins 
than that in core regions, it appears to be a possible reason for the presence of relatively larger amounts of $\varepsilon$-hcp phase in shell region as compared to that in the core regions. Figure 11 shows the variation of hardness of fine-grained shell and coarse grained core regions with increasing strain. It can be clearly noted that the hardness of both the regions increased with increasing applied strain and the average hardness of the fine-grained shell region was always significantly higher as compared to coarse-grained core region. However, it is interesting to note that fine-grained shell region exhibited a relatively higher rate of hardness increment in the initial stages of deformation whereas the coarse grained core region had relatively higher rate of increment in the later stages of deformation. It will be important to recall that the nominal stress-strain curves corresponding to harmonic structure also demonstrated the hardening behavior similar to fine-grained shell region. Therefore, these results clearly show that the inter-connected network of fine-grained shell region plays an important role in achieving higher strength in the harmonic structured materials due to its ability to undergo relatively higher strain hardening in the early stages of deformations, as compared to coarse-grained core regions.

The above results demonstrate that the harmonic structured Co-Cr-Mo alloy, with bimodal grain size distribution, demonstrates a very complex deformation behavior. It appears that fine-grained shell region dominates the deformation behavior, especially the higher strength and high strain hardening rates in the early stages of deformations, as relatively stronger interconnected fine-grained shell needs to be deformed to commence any plastic deformation. The larger flow stresses seems to be associated with the large stresses required to activate twinning mechanisms and/or the movement of dissociated dislocations in the fine-grained shell region. Furthermore, in case of 
fine-grained shell regions, the higher strain hardening rates to ensure stable plastic flow until fracture appears to be an outcome of the strong interactions between dislocations with limited mobility and pre-existing/strain-induced structural defects such as stacking faults, twins, and other dislocations. Since the fine-grained materials do not possess active slip deformation mode as strain producing mechanism, fracture occurs at relatively smaller strains. However, the harmonic structured Co-Cr-Mo alloy demonstrated significant ductility, which could be attributed to the presence of coarse-grained core regions in the microstructure [31].

Figure 12 shows the reduction in the cross-sectional area of the gauge area along the gauge-length of the tensile specimens having harmonic and coarse-grained structure. It can be clearly noticed that the reduction in the cross-sectional area of the specimens with harmonic structure was more or less uniform along the gauge length, suggesting uniform plastic deformation in the specimens. On the other hand, the reduction in the cross-sectional area of the coarse-grained IP specimens was not uniform, indicating localized plastic deformation in the specimens. These results clearly indicate that the harmonic structure design promotes uniformity of plastic deformation, leading to enhanced ductility by avoiding the localization of plastic strain in the early stages of deformation. In case of harmonic structured Ti-6Al-4V alloy, it was demonstrated that the relatively soft/ductile "core" regions undergo plastic deformation to adjust the shape change of the skeleton of interconnected network of fine-grained shell regions [31]. Since the ductile core is constrained inside the stronger shell region, it deforms in such a way that the strain incompatibilities at the core-shell interfaces and overall plastic deformation are accommodated without disrupting the continuum/integrity of the material. It was demonstrated that such a deformation mechanism delayed localization 
of plastic strain by promoting uniform distribution of strain during plastic deformation. As a result, larger deformation strains are achieved in harmonic structured material as compared to its homogeneous coarse/fine- grained counterparts.

\subsection{Effect of Bimodal Structure Topology on the Deformation Behavior and Mechanical Properties}

Figure 13 shows the representative microstructure and strain-strain curves corresponding to the specimens with harmonic and conventional heterogeneous bimodal structures. Both types of microstructure consisted of bimodal grain size distribution having approximately 60 volume $\%$ of fine grained area. However, despite of these similarities, these microstructures have different characteristics in terms of spatial distribution of fine-grained and coarse-grained areas. The harmonic structure has a peculiar distribution of fine- and coarse-grained areas wherein the coarse-grained areas are enclosed in a continuously connected three-dimensional network of fine-grained "walls". In contrast, the compact with conventional bimodal structure has an irregular heterogeneous distribution of coarse- and fine-grained areas wherein the fine-grained areas appear as isolated patches. Therefore, henceforth, this microstructure will be referred as "heterogeneous bimodal structure".

From Figure 13, it can also be noted that the tensile stress-strain curves of both the samples having bimodal grain size distribution exhibit more or less similar strength values. However, it is interesting to note that the specimens with harmonic structure exhibit considerably higher ductility as compared to those with heterogeneous bimodal structure. Figure 14 illustrates the reduction in cross-sectional area along the gauge of the tensile specimens having harmonic structure and heterogeneous bimodal structure. It 
can be clearly observed that, under similar level of plastic deformation, the specimen with harmonic structure resulted in more homogeneous reduction in the gauge region as compared to that of the specimen with "heterogeneous" bimodal structure. These results clearly indicate that the harmonic structure design promotes uniformity of deformation throughout the specimen under tensile loading. As a result, the localization of plastic deformation is delayed, leading to the shifting of the point of plastic instability to higher strain values.

The uniformity of plastic deformation and delayed localization of strain appears to be related with the complex modes of plastic deformation operating in the coarse-grained core region owing to the constraints applied by the fine-grained shell region of the harmonic structure due to its unique topology. It appears that such constraints lead to the deformation of the coarse-grained core region through a mixed mode of deformation consisting of shear, compressive, and tensile, rather than pure tensile mode. As a result, strain localization is avoided due to efficient strain accommodation via uniform strain distribution, leading to the extension of the effective strain hardening to a larger deformation range. On the other hand, heterogeneous bimodal structure does not provide any such constrained deformation to coarse-grained areas, leading to the possibility of strain localization in coarse-grained areas at relatively lower deformation under tensile loading. Consequently, harmonic structure demonstrates higher ductility and ultimate tensile strength as compared to heterogeneous bimodal structure.

Similar results have also been found in the case of harmonic structured SUS304L grade stainless steel wherein it was demonstrated that the harmonic structure may appear heterogeneous at the micro-scale, it is extremely effective in promoting the overall homogeneity of plastic deformation on macro-scale and delaying the localization of 
plastic deformation under tensile straining [29-30]. Furthermore, it was also demonstrated that, as compared to heterogeneous bimodal structure, the creation of harmonic structure increases not only the average values of both strength and ductility but also the reproducibility of results by reducing the range of variation in the mechanical properties.

\section{Conclusions}

Co-Cr-Mo alloy compacts with harmonic structure design, a unique microstructural design based on bimodal grain size distribution, were successfully prepared via a powder metallurgy route involving controlled mechanical milling and spark plasma sintering of pre-alloyed powders. The continuously-connected network of fine-grained shell region consisted of equiaxed grains (grain size: $2-5 \mu \mathrm{m}$ ) with a significant amount of annealing twins together with a small amount of $\varepsilon$-hcp martensitic phase. The specimens with harmonic structure exhibited relatively higher strength, both yield strength and tensile strength, together with higher ductility as compared to the coarse-grained specimens. It was also found that the specimens with harmonic structure and heterogeneous bimodal structure, with comparable volume fraction of fine-grained area, had similar strength values. However, the harmonic Co-Cr-Mo specimens exhibited considerably higher ductility as compared to those with heterogeneous bimodal structure. The "harmonic" Co-Cr-Mo alloy demonstrated a very complex deformation behavior wherein the fine-grained shell region was found to be more susceptible to undergo strain-induced $\alpha$-fcc to $\varepsilon$-hcp phase transformation as compared to the coarse-grained core regions, under a given plastic deformation, due to the presence of higher number of annealing twins together with deformation induced other 
structural defects. Subsequently, the deformation behavior demonstrated that the higher strength and high retained ductility are derived from fine-grained shell and coarse-grained core regions, respectively. Finally, it was demonstrated that the peculiar topological distribution of strong fine-grained and ductile coarse-grained regions in the harmonic structure promotes uniform distribution of strain, resulting in improved mechanical properties by suppressing the localized plastic deformation during straining.

\section{Acknowledgement}

This research was supported by the Japan Science and Technology Agency (JST) under Collaborative Research Based on Industrial Demand "Heterogeneous Structure Control: Towards Innovative Development of Metallic Structural Materials", and by the Grant-in-Aid for Scientific Research on Innovative Area, "Bulk Nanostructured Metals", through MEXT, Japan (contract No. 22102004). These supports are gratefully appreciated.

\section{$\underline{\text { References }}$}

[1] L. Shi, D. O. Northwood, Z. Cao, Alloy design and microstructure of a biomedical Co-Cr alloy, J. Mater. Sci. 28 (1993) 1312- 1316.

[2] G. Mani, M.D. Feldman, D. Patel, C.M. Agarwal, Coronary stents: A materials perspective, Biomaterials 28 (2007) 1689-1710.

[3] R.B. Frank, K.A. Heck, J. Stravinskas, Beryllium-free Cobalt Alloy for High-load Bushings, Adv. Mater. Proces. March (2010) 19-23.

[4] H. Gleiter, Nanostructured Materials: Basic Concepts and Microstructure, Acta Mater. 48 (2000) 1-29.

[5] N. Hansen, Hall-Petch relation and boundary strengthening, Scr. Mater. 51(2004)801-806. 
[6] M.A. Meyers, A. Mishra, D.J. Benson, Mechanical properties of nanocrystalline materials, Prog. Mater. 51 (2006) 427-556.

[7] R.Z. Valiev, M.J. Zehetbauer, Y. Estrin, H.W. Hoppel, Y. Ivanisenko, H. Hahn, G. Wilde, H.J. Roven, X. Sauvage, T.G. Langdon, The Innovation Potential of Bulk Nanostructured Materials, Adv. Eng. Mater. 9 (2007) 527-533.

[8] Y. Estrin, A. Vinogradov, Extreme grain refinement by severe plastic deformation: A wealth of challenging science, Acta Mater. 61 (2013) 782-817.

[9] X. Sauvage, G. Wilde, S.V. Divinski, Z. Horita, R.Z. Valiev, Grain boundaries in ultrafine grained materials processed by severe plastic deformation and related phenomena, Mater. Sci. Eng. A 540 (2012) 1-12.

[10] K. Yamanaka, M. Mori, A. Chiba, Mechanical properties of as-forged Ni-free Co-29Cr-6Mo alloys with ultrafine-grained microstructure, Mater. Sci. Eng. A 528 (2011) 5961-5966.

[11] M. Mori, K. Yamanaka, S. Sato, K. Wagatsuma, A. Chiba, Microstructures and Mechanical Properties of Biomedical Co-29Cr-6Mo-0.14N Alloys Processed by Hot Rolling, Metall. Mater. Trans. A 43 (2012) 3108-3119.

[12] K. Yamanaka, M. Mori, A. Chiba, Enhanced Mechanical Properties of As-Forged Co-Cr-Mo-N Alloys with Ultrafine-Grained Structures, Metall. Mater. Trans. A 43 (2012) 5243-5257.

[13] H. Jin, D.J. Lloyd, Effect of a duplex grain size on the tensile ductility of an ultra-fine grained Al-Mg alloy, AA5754, produced by asymmetric rolling and annealing, Scr. Mater. 50 (2004) 1319-1323.

[14] B. Srinivasrao, K. Oh-ishi, T. Ohkubo, T. Mukai, K. Hono, Synthesis of high-strength bimodally grained iron by mechanical alloying and spark plasma sintering, 
Scr. Mater. 58 (2008) 759-762.

[15] H.W. Hoppel, M. Korn, R. Lapovok, H. Mughrabi, Bimodal grain size distribution in UFG materials produced by SPD: Their evolution and effect on mechanical properties, J. Phys. Conf. Ser. 240 (2010) 012147.

[16] S. Zherebtsov, E. Kudryavtsev, S. Kostjuchenko. S. Malysheva, G. Salishchev, Strength and ductility-related properties of ultrafine grained two-phase titanium alloy produced by warm multiaxial forging, Mater. Sci. Eng. A 536 (2012) 190-196.

[17] S. Joshi, K. Ramesh, B. Han, E. Lavernia, Modeling the Constitutive Response of Bimodal Metals, Metall. Mater. Trans. A 37 (2006) 2397-2404.

[18] Y. Wang, M. Chen, F. Zhou, E. Ma, High tensile ductility in a nanostructured metal, Nature 419 (2002) 912-915.

[19] D. Witkin, Z. Lee, R. Rodriguez, S. Nutt, E. Lavernia, Al-Mg alloy engineered with bimodal grain size for high strength and increased ductility, Scr. Mater. 49 (2003) 297-302.

[20] G.J. Fan, H. Choo, P.K. Liaw, E.J. Lavernia, Plastic deformation and fracture of ultrafine-grained Al-Mg alloys with a bimodal grain size distribution, Acta Mater. 54 (2006) 1759-1766.

[21] Z. Lee, V. Radmilovic, B. Ahn, E.J. Lavernia, S.R. Nutt, Tensile Deformation and Fracture Mechanism of Bulk Bimodal Ultrafine-Grained Al-Mg Alloy, Metall. Mater. Trans. A 41 (2010) 795-801.

[22] G. Dirras, J. Gubicza, S. Ramtani, Q.H. Bui, T. Szilagyi, Microstructure amd mechanical characteristics of bulk polycrystalline $\mathrm{Ni}$ consolidated from blends of powders with different particle size, Mater. Sci. Eng. A 527 (2010) 1206-1214.

[23] G. Dirras, D. Tingaud, G. Csiszar, J. Gubicza, H. Couque, F. Mompiou, 
Characterization of bulk bimodal polycrystalline nickel deformed by direct impact loadings, Mater. Sci. Eng. A 601 (2014) 48-57.

[24] H. Fujiwara, R. Akada, A. Noro, Y. Yoshita, K. Ameyama, Enhanced Mechanical Properties of Nano/Meso Hybrid Structure Materials Produced by Hot Roll Sintering Process, Mater. Trans. 49 (2008) 90-96.

[25] H. Fujiwara, T. Sekiguchi, K. Ameyama, Mechanical properties of pure titanium and Ti-6Al-4V alloys with a new tailored nano/meso hybrid microstructure, Int. J. Mater. Res. 6 (2009) 796-799.

[26] T. Sekiguchi, K. Ono, H. Fujiwara, K. Ameyama, New Microstructure Design for Commercially Pure Titanium with Outstanding Mechanical Properties by Mechanical Milling and Hot Roll Sintering, Mater. Trans. 51 (2010) 39-45.

[27] D. Orlov, H. Fujiwara, K. Ameyama, Obtaining Copper with Harmonic Structure for the Optimal Balance of Structure-Performance Relationship, Mater. Trans. 54 (2013) $1549-1553$.

[28] O.P. Ciuca, M. Ota, S. Deng, K. Ameyama, Harmonic Structure Design of a SUS329J1 Two Phase Stainless Steel and Its Mechanical Properties, Mater. Trans. 54 (2013) 1629-1633.

[29] Z. Zhang, S.K. Vajpai, D. Orlov, K. Ameyama, Improvement of mechanical properties in SUS304L steel through the control of bimodal microstructure characteristics, Mater. Sci. Eng. A 598 (2014) 106-113.

[30] Z. Zhang, D. Orlov, S.K. Vajpai, B. Tong, K. Ameyama, Importance of Bimodal Structure Topology in the Control of Mechanical Properties of a Stainless Steel, Adv. Eng. Mater. 2015, doi. 10.1002/adem.201400358.

[31] S.K. Vajpai, M. Ota, T. Watanabe, R. Maeda, T. Sekiguchi, T. Kusaka, K. Ameyama, 
The Development of High Performance Ti-6Al-4V Alloy via a Unique Microstructural Design with Bimodal Grain Size Distribution, Metall. Mater. Trans. A 46 (2015) 903-914.

[32] C. Sawangrat, O. Yamaguchi, S.K. Vajpai, K. Ameyama, Application of Harmonic Structure Design to Biomedical Co-Cr-Mo Alloy for Improved Mechanical Properties, Mater. Trans. 55(2014) 99-105.

[33] M. Sage, C. Gillaud, Methode d'analyse quantitative des varietes allotropiques du cobalt par les rayons X, Rev. Metall. 49 (1950) 139-145.

[34] G.B. Olson, M. Cohen, A General Mechanism of Martensitic Nucleation: Part III. Kinetics of Martensitic Nucleation, Metall. Trans. A 7A (1976) 1915-1923.

[35] P. Huang, H.F. Lopez, Strain induced $\varepsilon$-martensite in a Co-Cr-Mo alloy: grain size effects, Mater. Lett. 39 (1999) 244-248.

[36] A. Salinas-Rodriguez, J.L. Rodriguez-Galicia, Deformation behavior of low-carbon Co-Cr-Mo alloys for low-friction implant applications, J. Biomed. Mater. Res. 31 (1996) 409-419.

[37] A. Mani, Salinas-Rodriguez, H.F. Lopez, Deformation induced FCC to HCP transformation in a Co-27Cr-5Mo-0.05C alloy, Mater. Sci. Eng. A 528 (2011) 3037-3043.

[38] H.R. Lashgari, Sh. Zangeneh, F. Hasanabadi, M. Saghafi, Microstructural evolution during isothermal aging and strain-induced transformation followed by isothermal aging in Co-Cr-Mo-C alloy: A comparative study, Mater. Sci. Eng. A 527 (2010) 4082-4091.

[39] L. Remy, A. Pineau, Twinning and Strain-Induced F.C.C. $\rightarrow$ H.C.P. Transformation on the Mechanical Properties of Co-Ni-Cr-Mo Alloys, Mater. Sci. Eng. 26 (1976) 123-132. 
[40] X. Wu, N. Tao, Y. Hong, G. Liu, B. Xu, J. Lu, K. Lu, Strain-induced grain refinement of cobalt during surface mechanical attrition treatment, Acta. Mater. 53 (2005) 681-691.

[41] B.S. Lee, H. Matsumoto, A. Chiba, Fractures in tensile deformation of biomedical Co-Cr-Mo-N alloys, Mater. Lett. 65 (2011) 843-846.

[42] Y. Koizumi, S. Suzuki, K. Yamanaka, B. Lee, K. Sato, Y. Li, S. Kurosu, H. Matsumoto, A. Chiba, Strain-induced martensitic transformation near twin boundaries in a biomedical Co-Cr-Mo alloy with negative stacking fault energy, Acta Mater. 61(2013) 1648-1661.

\section{$\underline{\text { Figure Captions }}$}

Figure 1. A Schematic illustrating the Harmonic Structure Design and the proposed powder metallurgy method.

Figure 2. Microstructure of the sintered Co-Cr-Mo alloy compacts prepared from milled PREP powders.

Figure 3. XRD pattern of the sintered Co-Cr-Mo alloy compacts prepared from milled PREP powders.

Figure 4. (a) Microstructure (EBSD-IQ) and (b) corresponding phase distribution map, illustrating the core-shell area of the harmonic structured Co-Cr-Mo.

Figure 5. Representative nominal stress-strain curves of the coarse-grained IP and harmonic compacts.

Figure 6. Enlarged view of the initial part of the nominal stress-strain curves of the initial coarse-grained IP and harmonic compacts. 
Figure 7. XRD patterns of harmonic Co-Cr-Mo alloy showing microstructural evolution with increasing degree of deformation.

Figure 8. Variation of $\varepsilon$-hcp with increasing plastic strain in the harmonic structured and coarse-grained IP Co-Cr-Mo alloys.

Figure 9. Distribution of $\varepsilon$-hcp phase with increasing plastic strain in the harmonic structured Co-Cr-Mo alloys: (a), (b), and (c) EBSD-GB image after 0\%, 10\%, and 20\% deformation, respectively; (d), (e), and (f) phase distribution map of the area corresponding to (a), (b), and (c), respectively.

Figure 10. (a) Microstructure, and (b) phase distribution map, of the deformed ( 20\%) harmonic Co-Cr-Mo alloy. The intra-granular striations are marked by arrows.

Figure 11. Variation of hardness of fine-grained shell and coarse-grained core regions with increasing strain.

Figure 12: Reduction in the cross-sectional area of the gauge area along the gauge-length of the tensile specimens having harmonic and coarse-grained structure.

Figure 13. The representative microstructure and strain-strain curves corresponding to the specimens with harmonic structure and conventional "heterogeneous bimodal structure".

Figure 14. The reduction in cross-sectional area along the gauge of tensile specimens with harmonic structure and heterogeneous bimodal structure. 
Figure 1

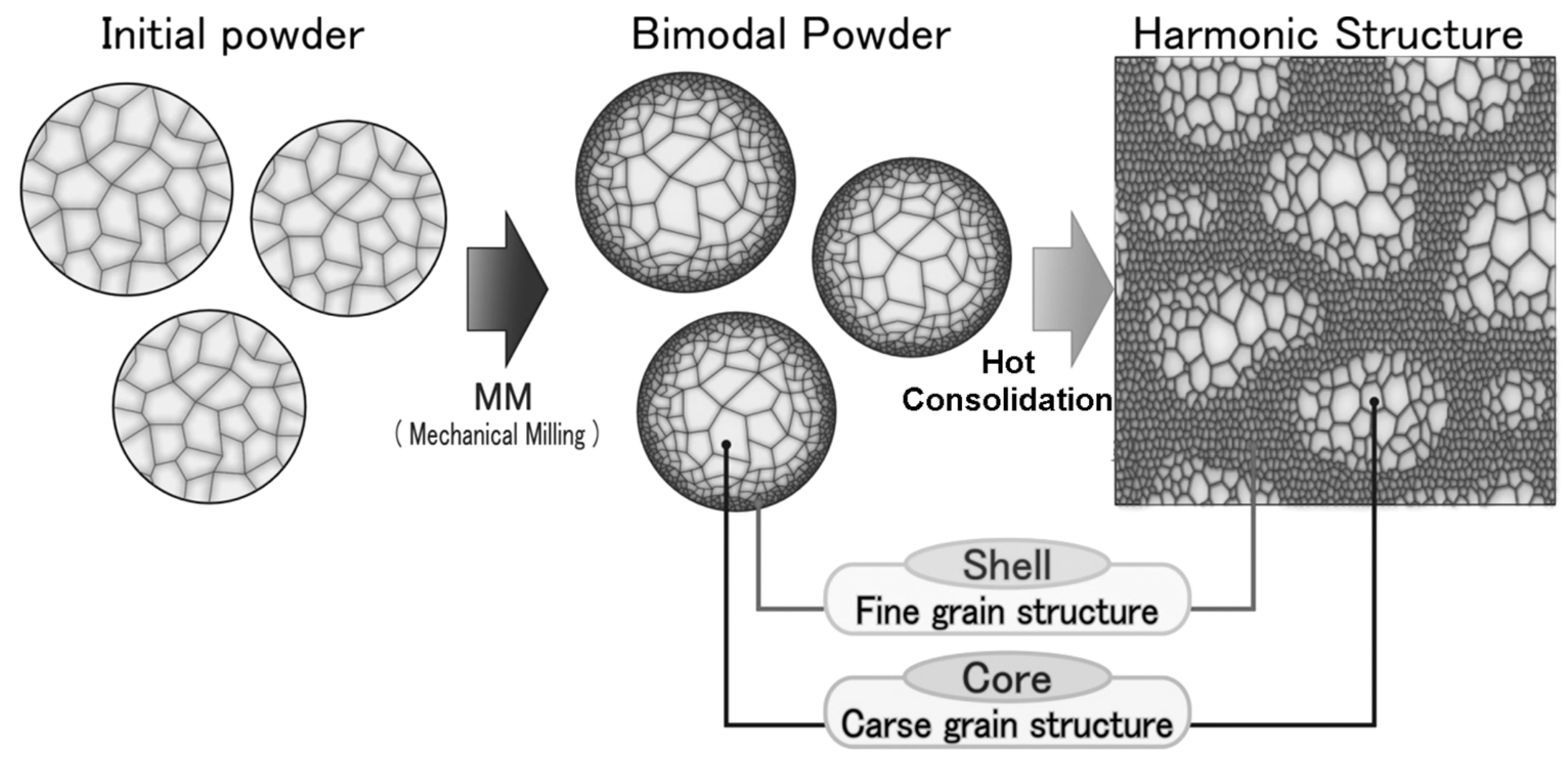




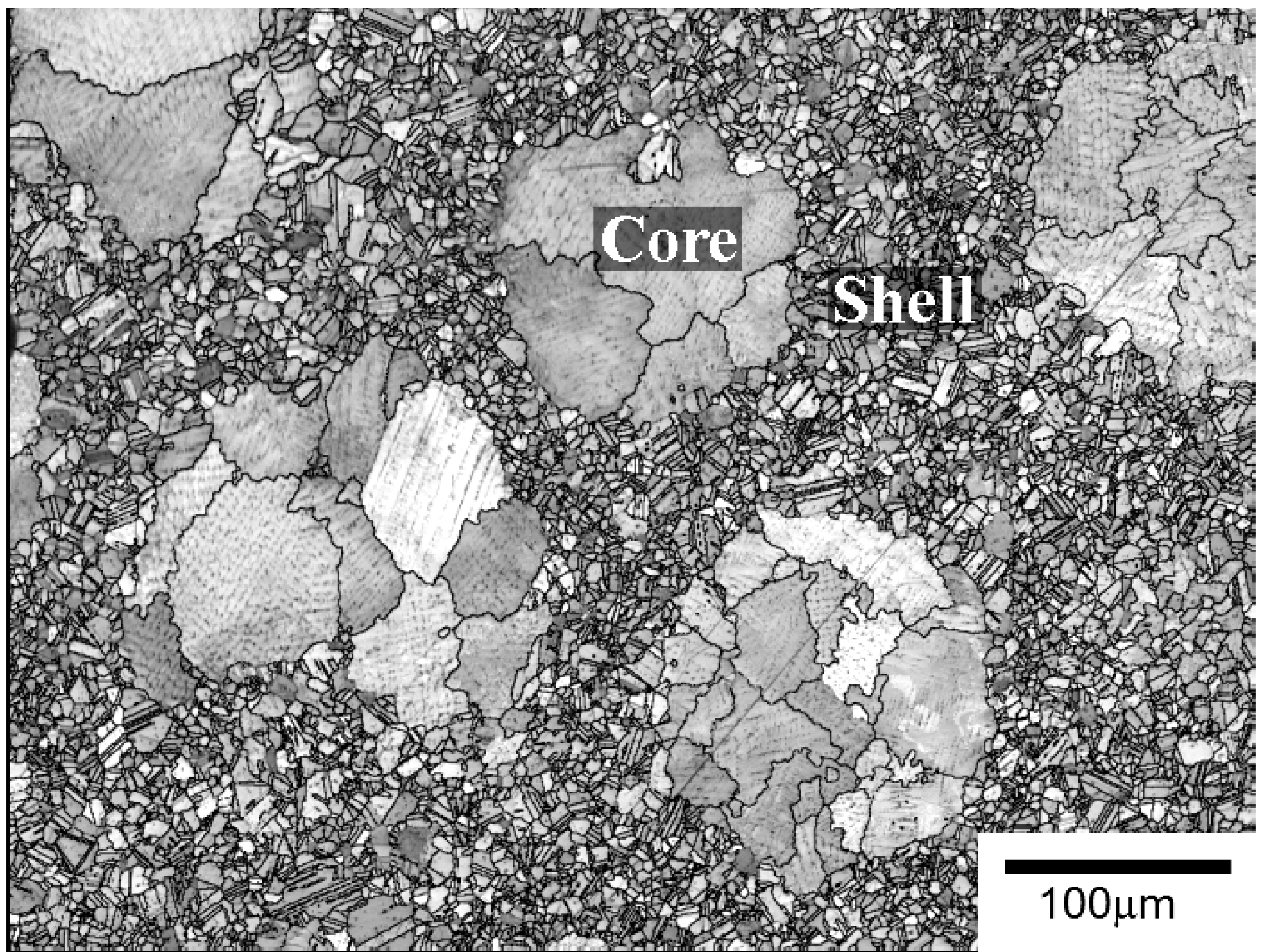




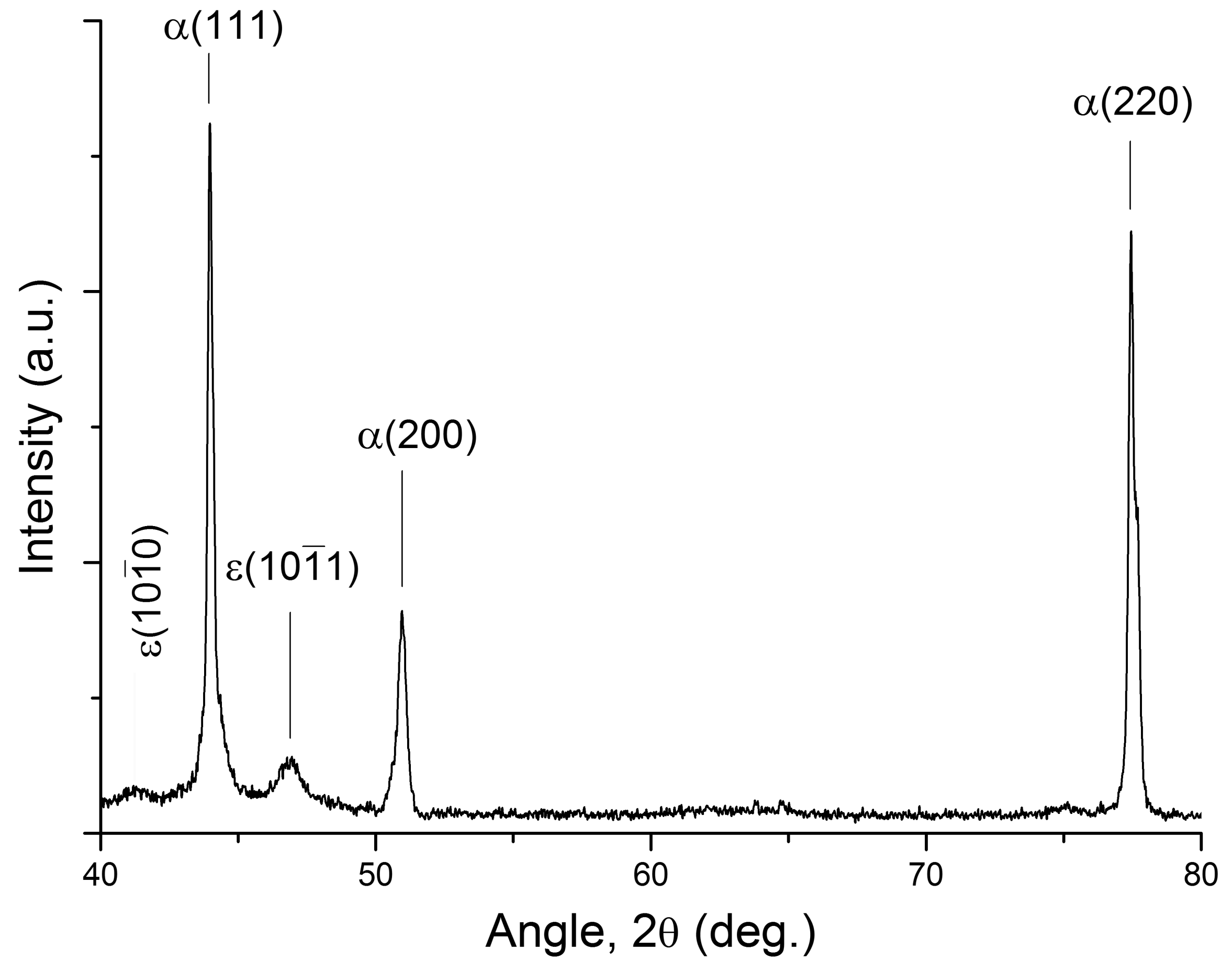




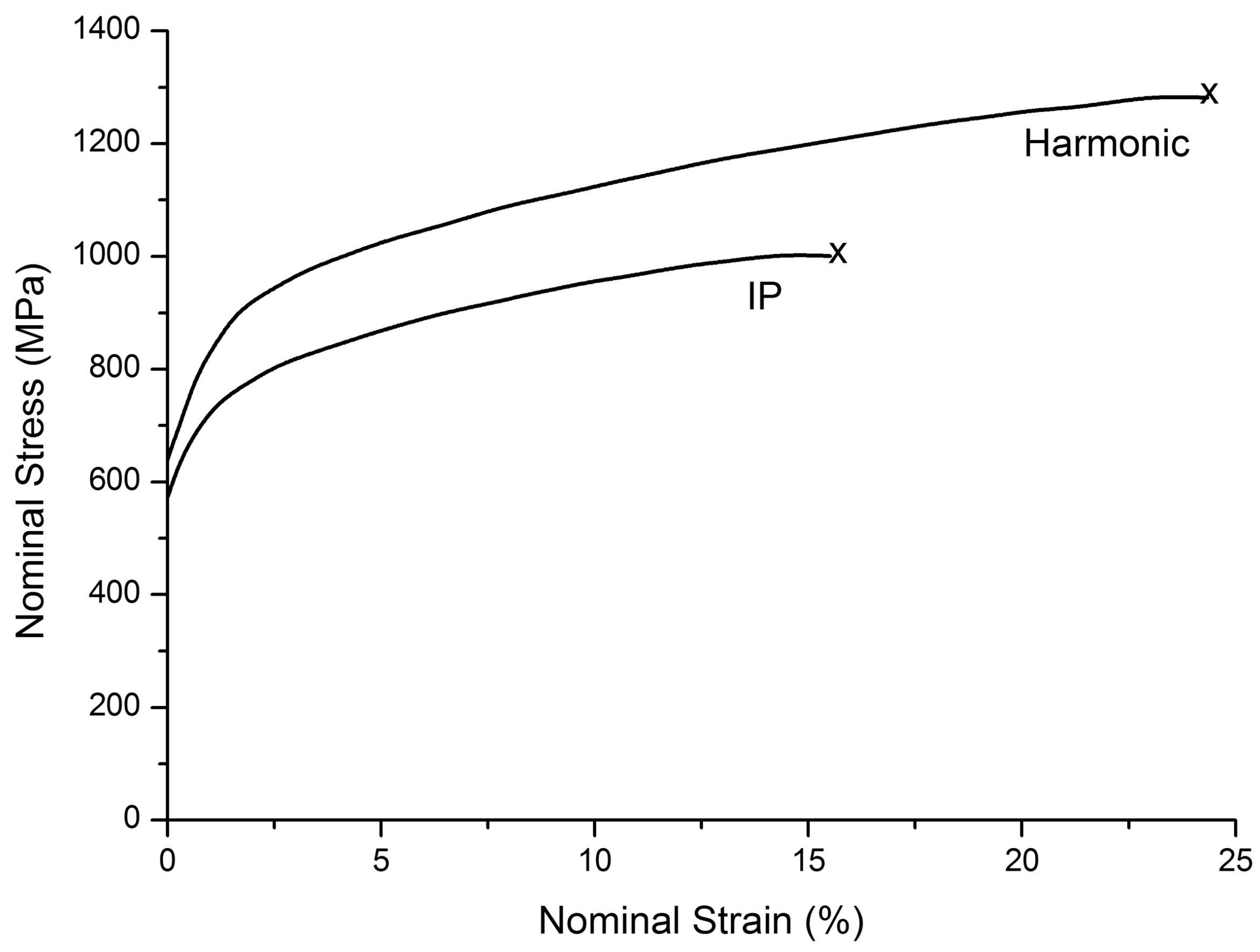




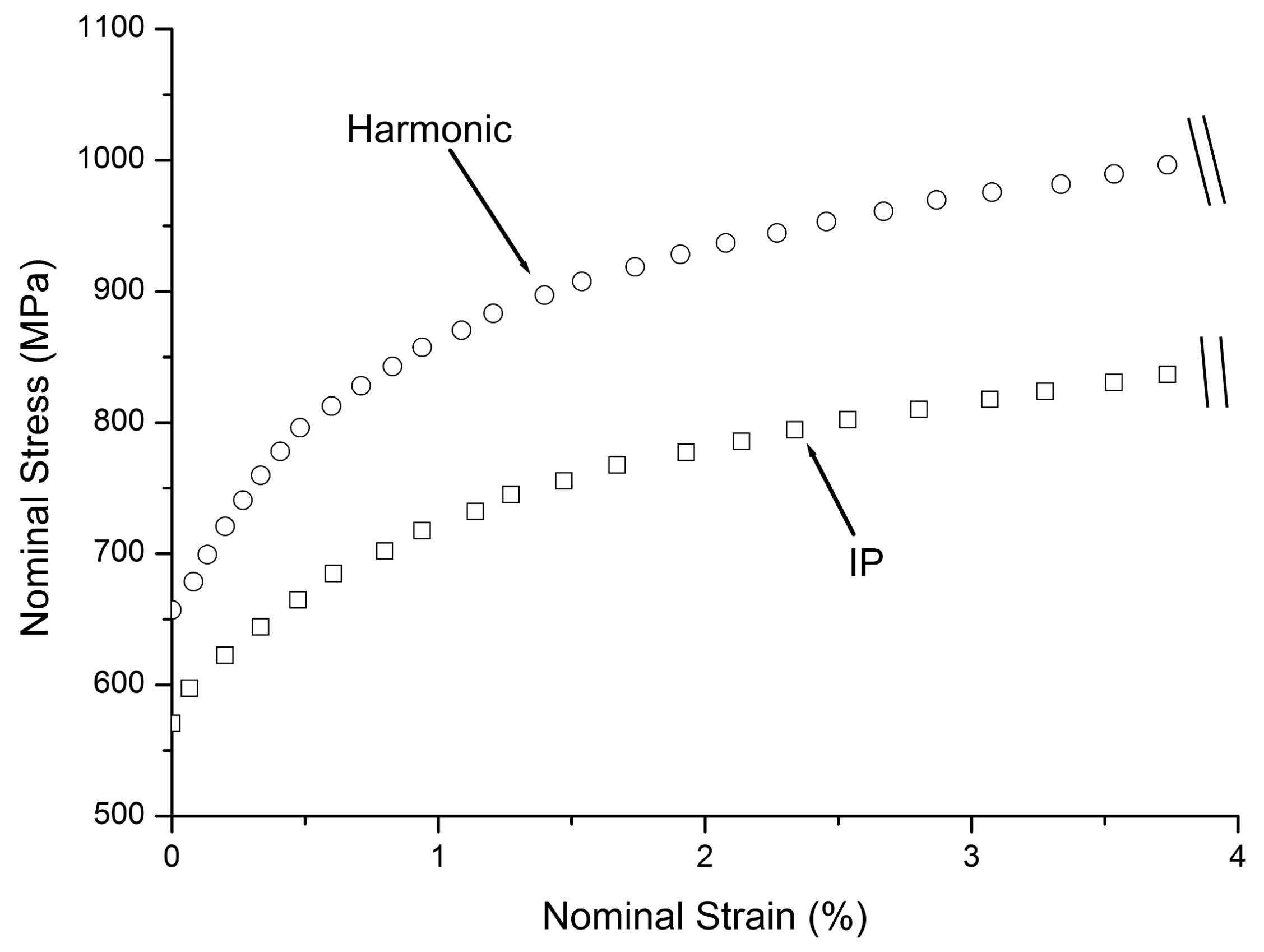




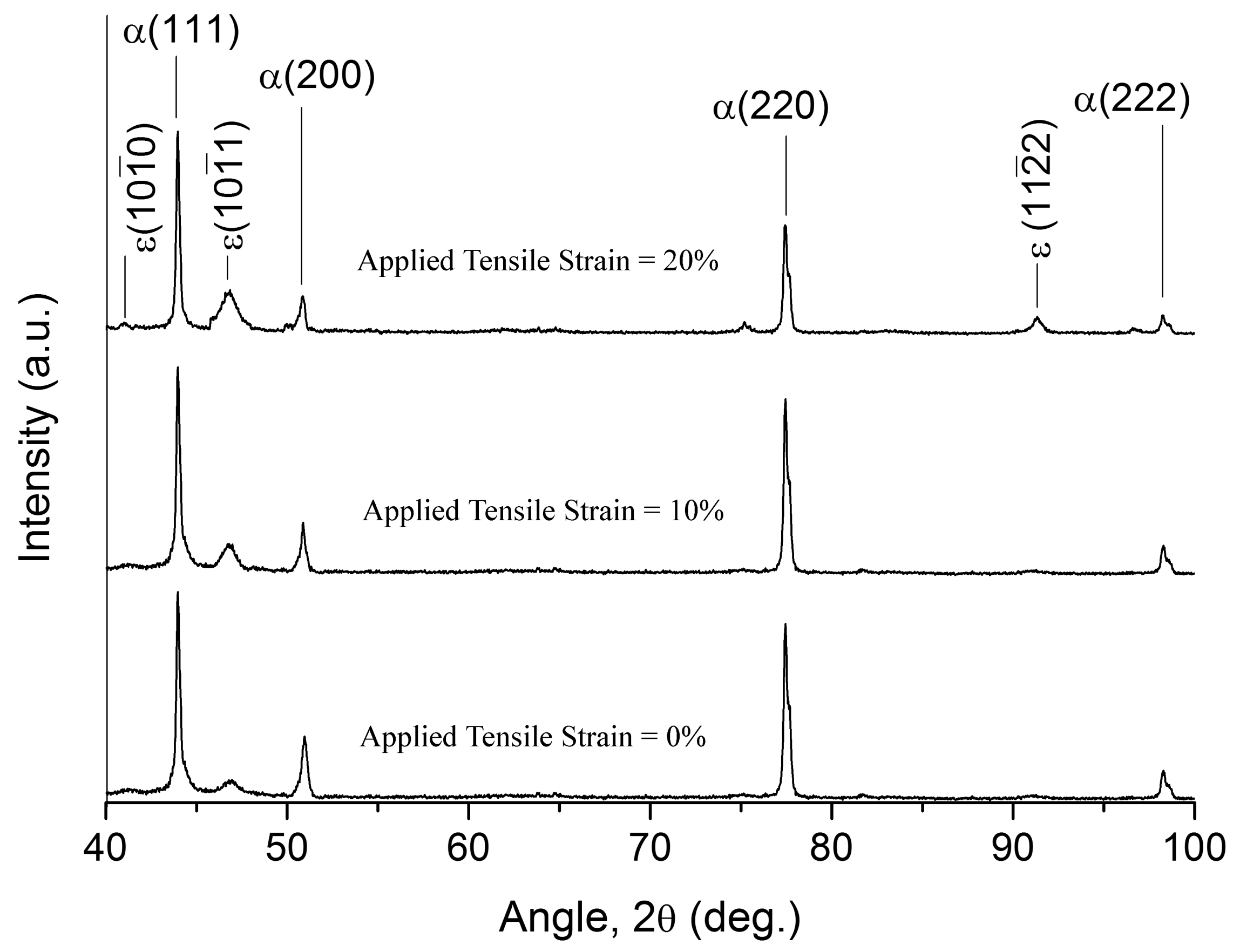




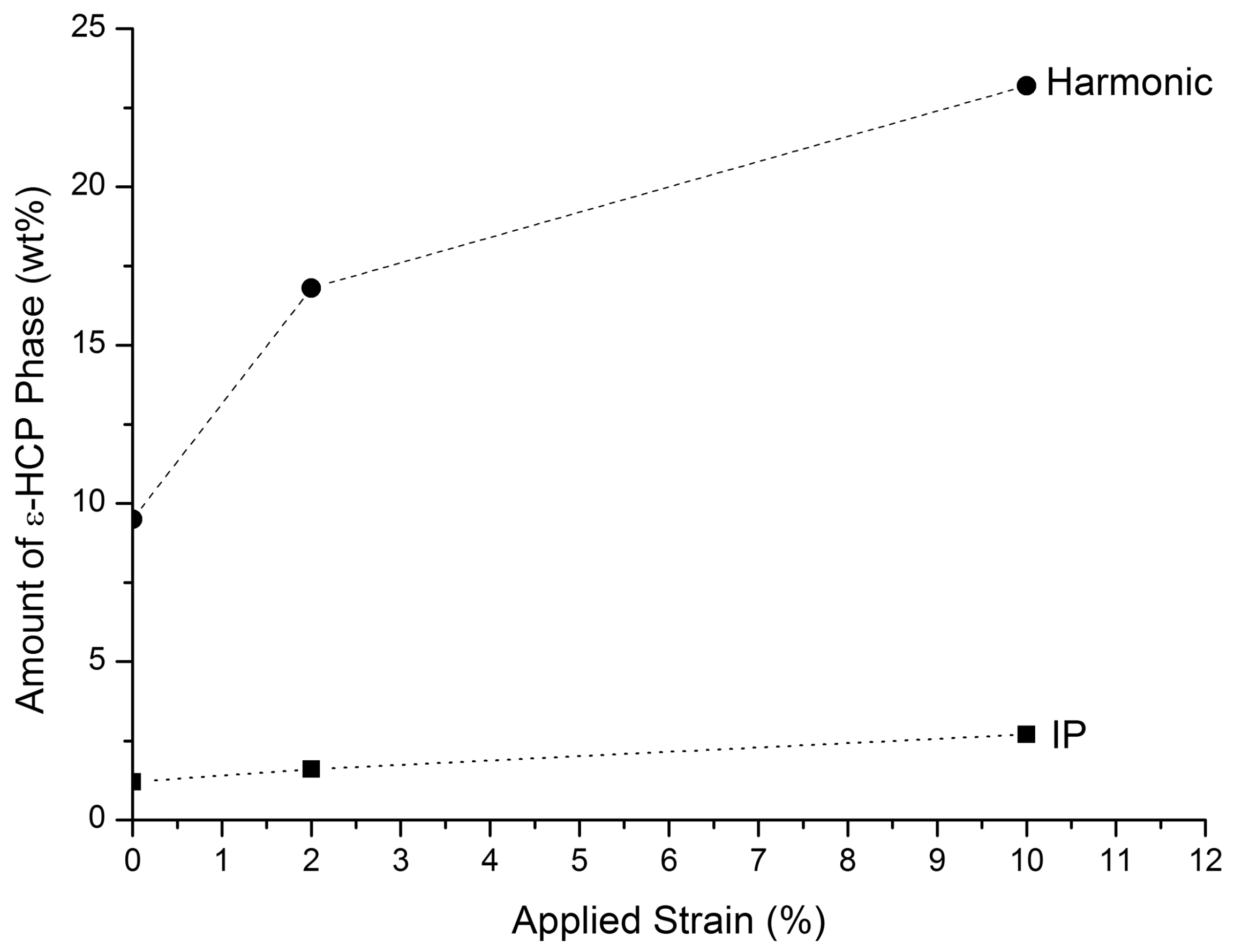




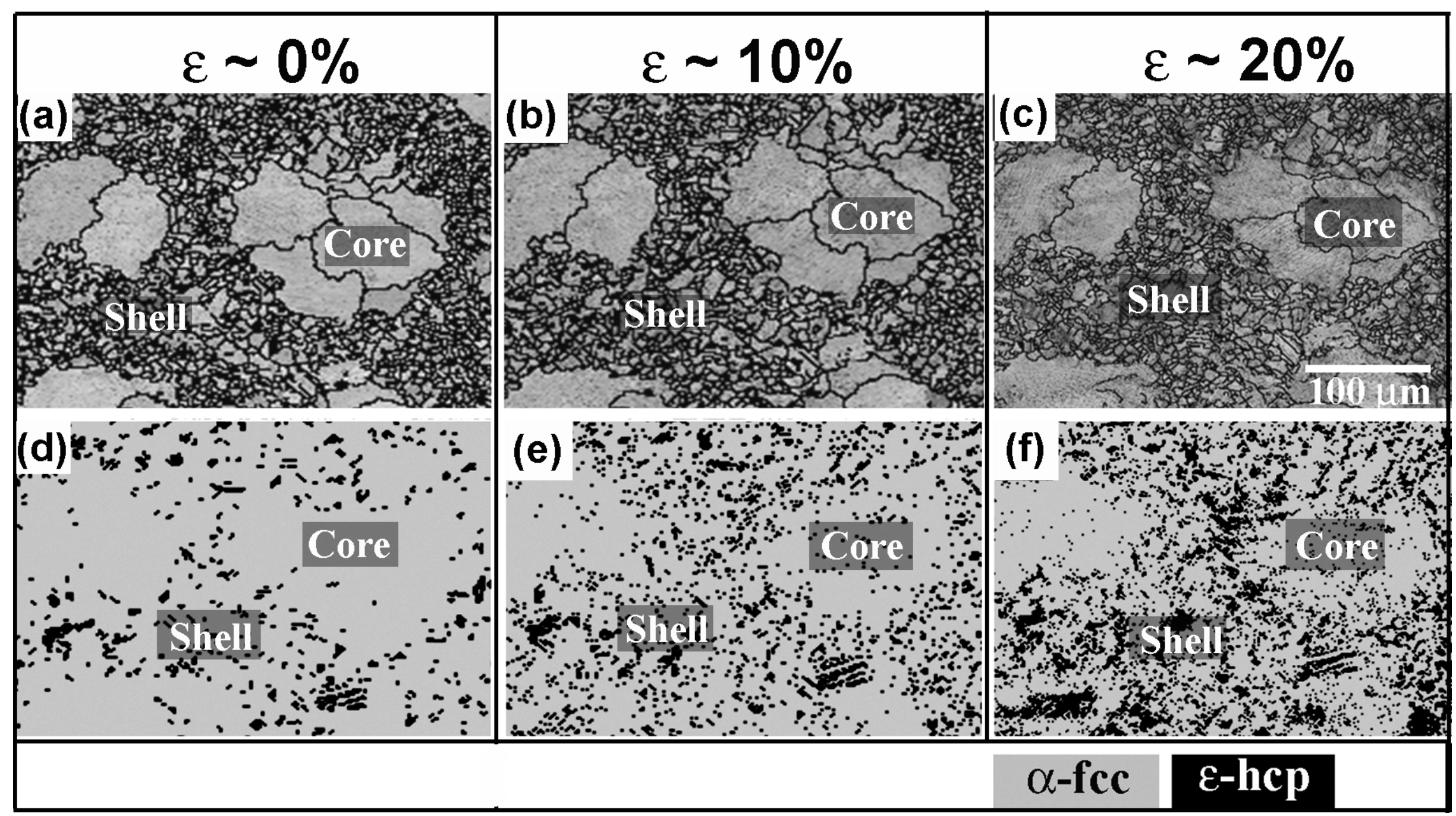



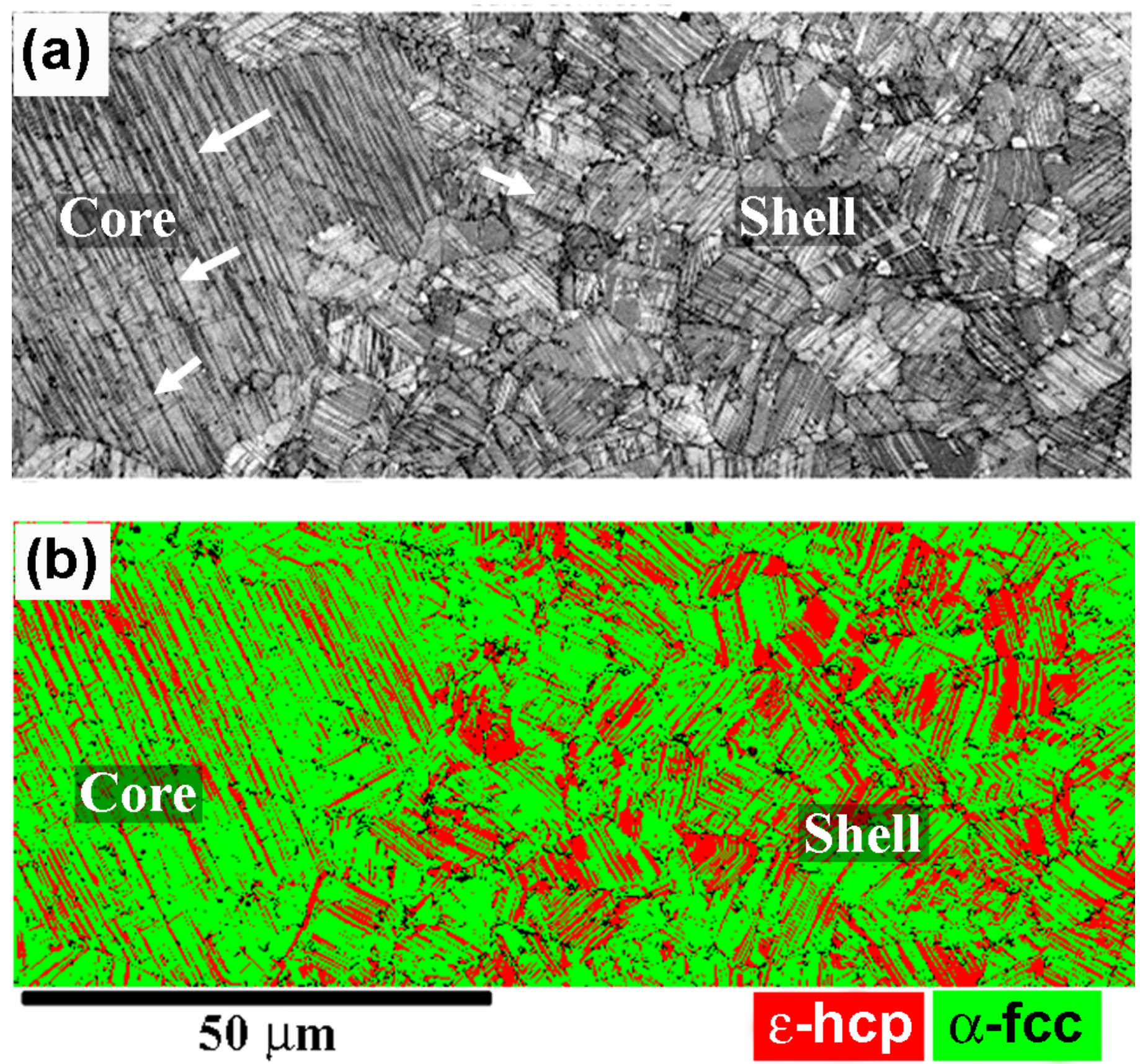
Figure 11

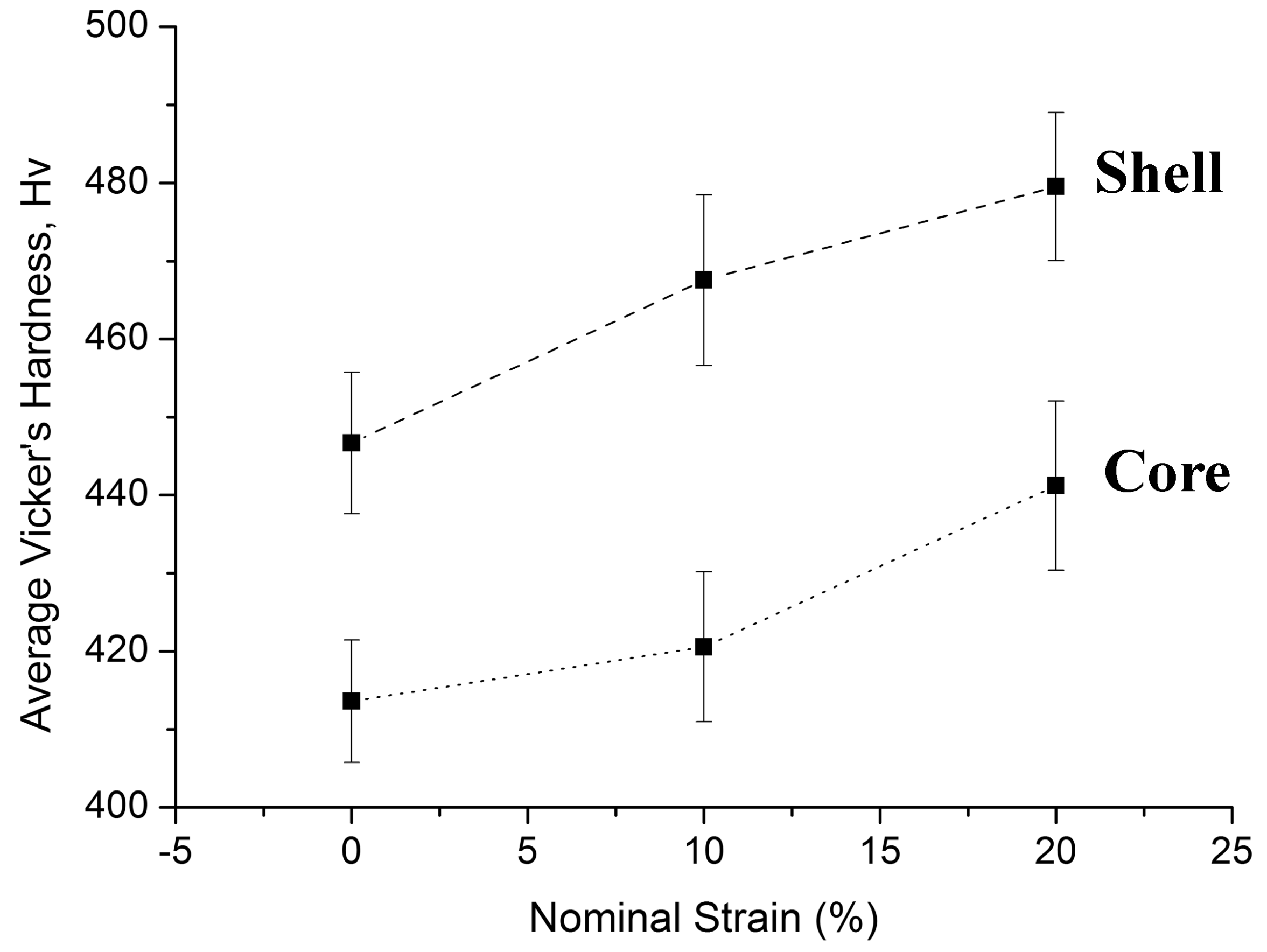




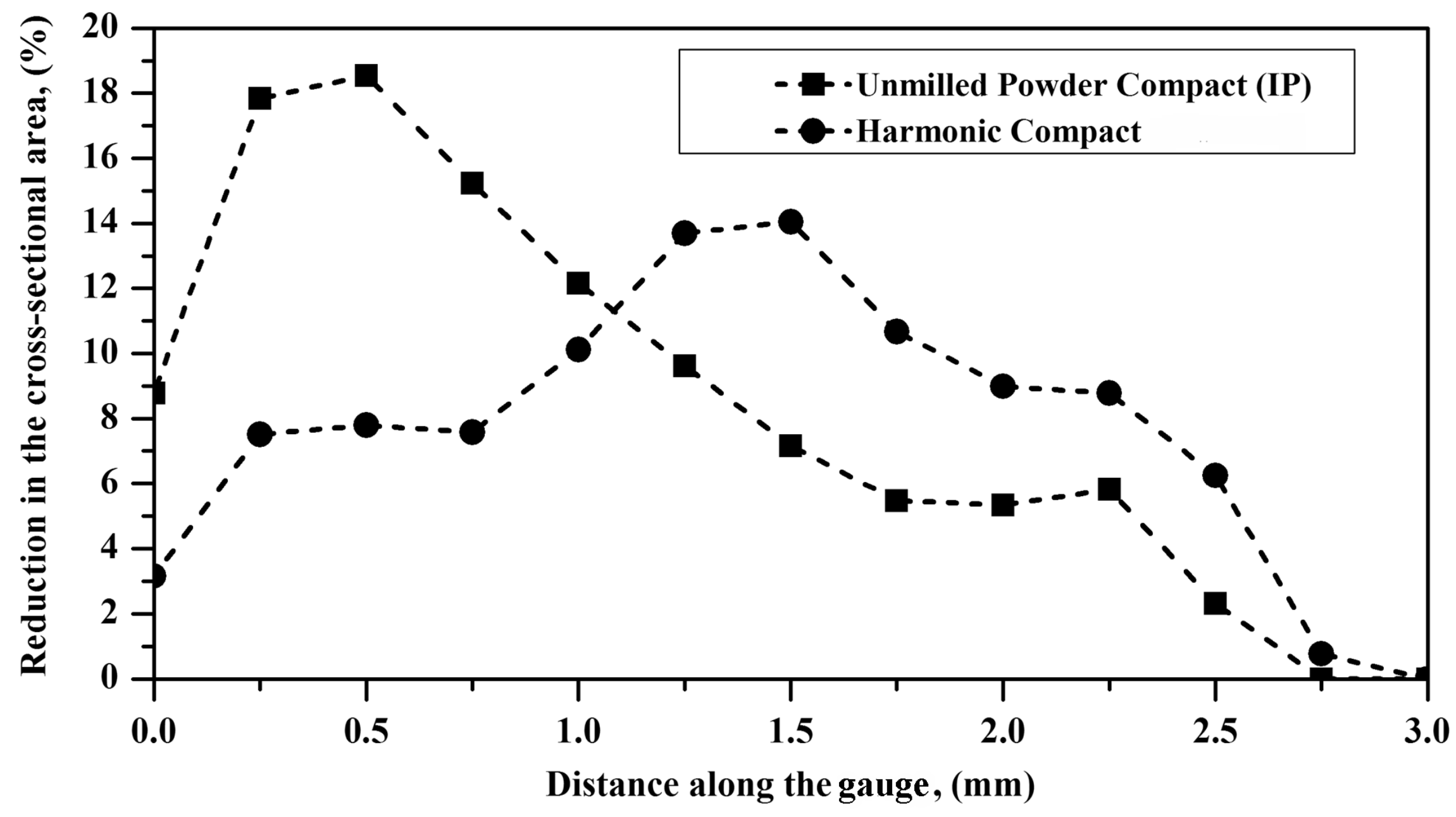


Figure 13

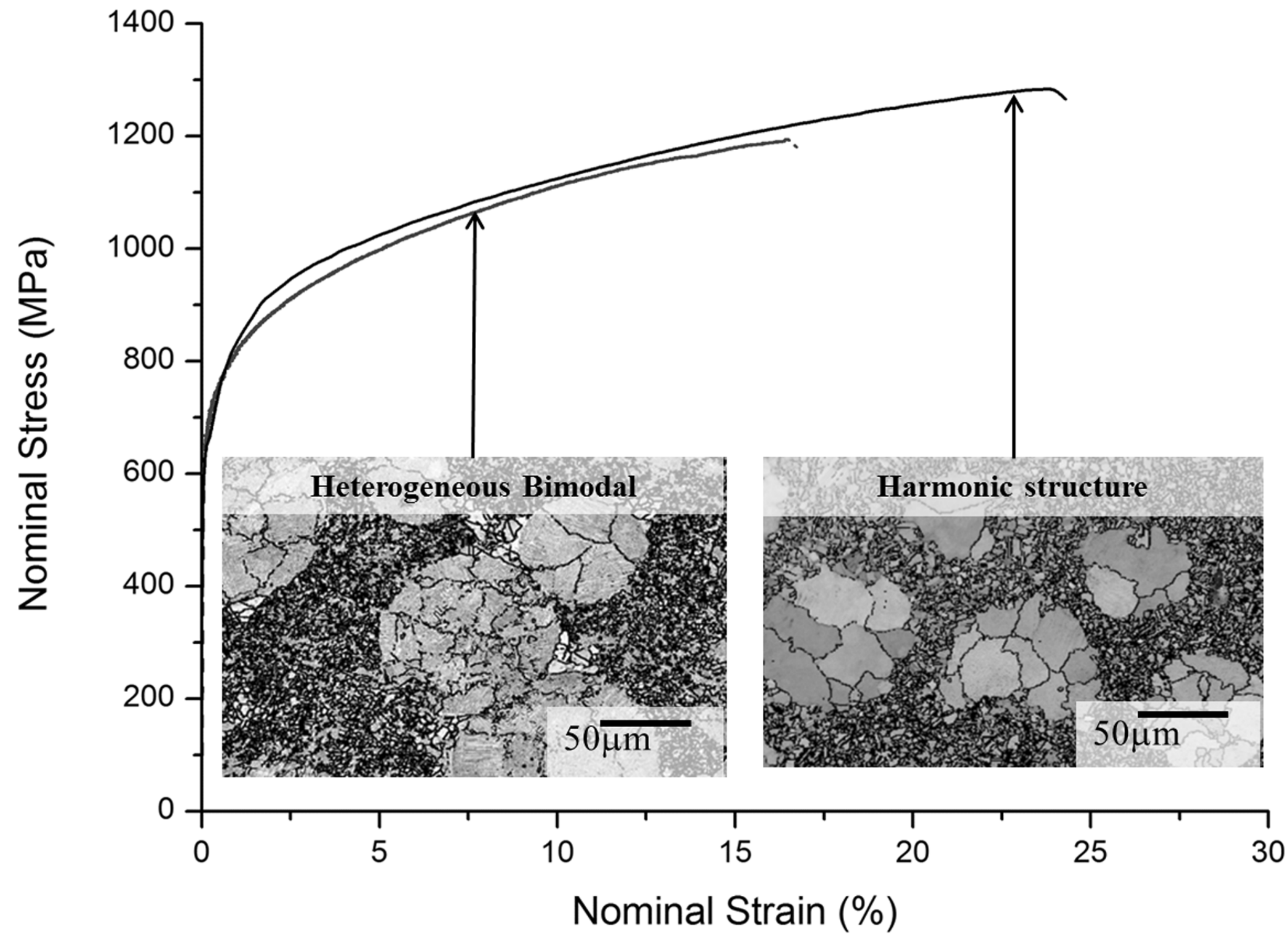




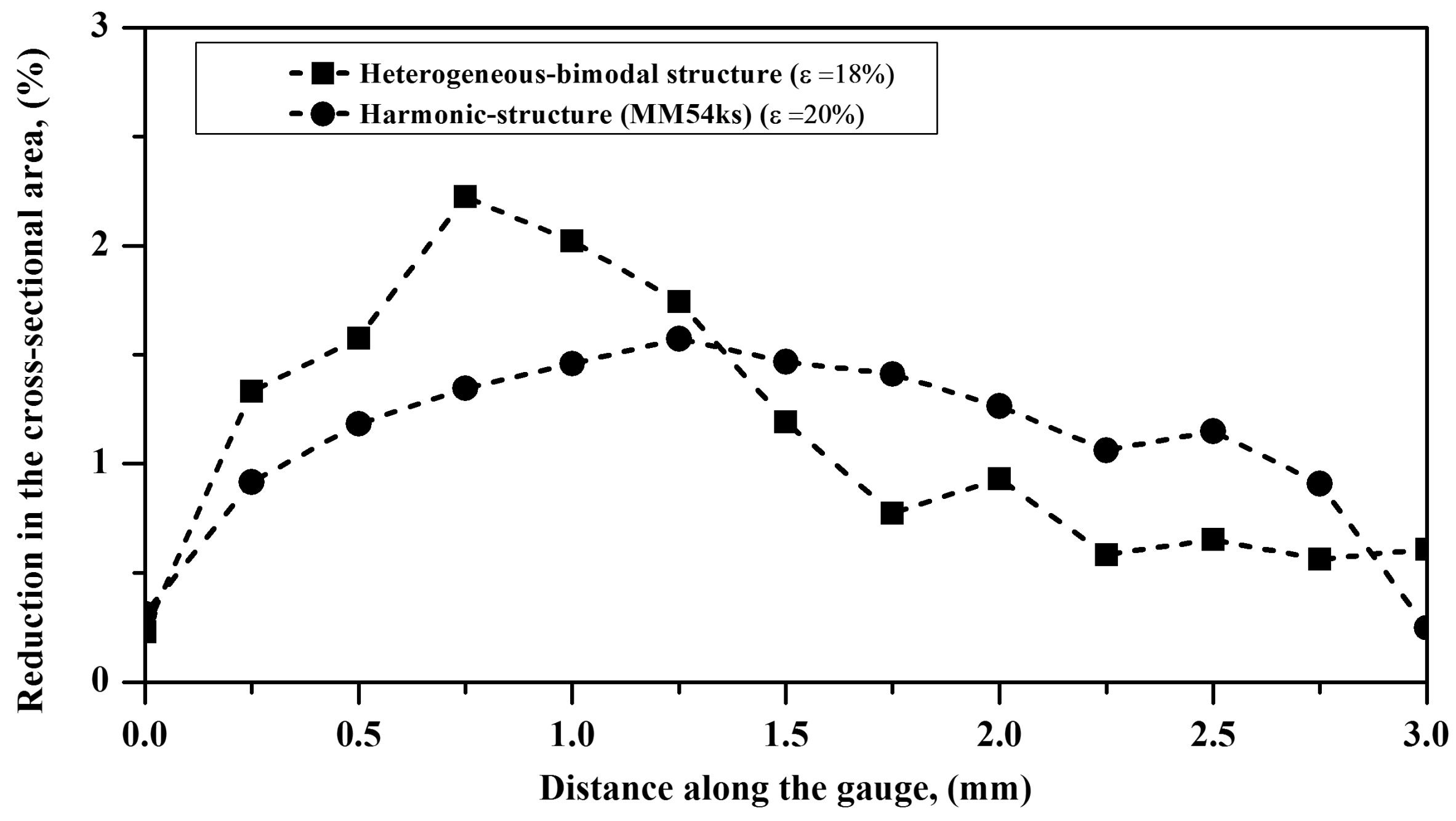


Table 1: Chemical composition of the PREP Co-Cr-Mo alloy powders.

\begin{tabular}{|l|l|l|l|l|l|l|l|l|}
\hline Element & $\mathrm{Cr}$ & $\mathrm{Mo}$ & $\mathrm{Ni}$ & $\mathrm{Fe}$ & $\mathrm{Mn}$ & $\mathrm{Si}$ & $\mathrm{C}$ & $\mathrm{Co}$ \\
\hline $\begin{array}{l}\text { Amount } \\
(\mathrm{mass} \%)\end{array}$ & 27.73 & 5.71 & 0.02 & 0.1 & 0.02 & 0.02 & 0.02 & Bal. \\
\hline
\end{tabular}

